\section{Cognitive inflexibility con
to both externalising and internalising difficulties in ASD}

\section{By Dr. Jessica Edwards}

Children with autism spectrum disorder (ASD) commonly experience internalising and externalising symptoms, but the underlying cognitive mechanisms are unclear. In their latest study published in the Journal of Child Psychology and Psychiatry, Ann Ozsivadjian and colleagues examined the role of three cognitive factors that might contribute to these difficulties. Specifically, they hypothesized that intolerance of uncertainty (IU) together with alexithymia might lead to internalising symptoms while cognitive inflexibility $(\mathrm{Cl})$ might lead to externalising behaviours and indirectly contribute to internalising symptoms via increasing IU.

To test their hypotheses, Ozsivadjian et al. recruited a sample of 95 5-18-year-olds with a clinical diagnosis of ASD and asked caregivers to complete questionnaires on ASD symptoms, internalising and externalising difficulties, $\mathrm{Cl}$, IU and alexithymia. They then analysed the relationships between these measures. In support of their hypothesis, they found that $\mathrm{Cl}$ predicted externalising symptoms directly and contributed to internalising problems indirectly via IU. Meanwhile, IU was directly related to internalising but not externalising problems, and alexithymia contributed to internalising problems only via IU.

The finding that $\mathrm{Cl}$ is involved in both externalising and internalising difficulties in children with ASD suggests that addressing $\mathrm{Cl}$ could have wide-ranging benefits. As such, the researchers propose that targeting $\mathrm{Cl}$ might be more beneficial than standard ASD-adapted cognitive behavioural approaches that tackle internalising or externalising symptoms separately.

\section{Referring to:}

Ozsivadjian, A., Hollocks, M.J., Magiati, I., Happe, F., Baird, G. \& Absoud, M. (2020), Is cognitive inflexibility a missing link? The role of cognitive inflexibility, alexithymia and intolerance of uncertainty in externalising and internalising behaviours in young people with autism spectrum disorder. J. Child Psychol. Psychiatr. doi: 10.1111/jcpp.13295.

\section{Glossary:}

Alexithymia: difficulty identifying and describing one's own emotions.

Cognitive inflexibility: the tendency to focus on one's own thoughts, beliefs or activity/behaviours with difficulty moving on to other thoughts and behaviours.

Internalising symptoms: emotional symptoms which tend to be expressed internally, such as anxiety and low mood.

Intolerance of uncertainty: the tendency to have a negative perception, interpretation, or experience of uncertainty.

Externalising symptoms: maladaptive behaviours which are directed externally towards the environment, such as aggressive behaviours and impulsivity. 